\title{
Attention-deficit Hyperactivity Disorder and Gluten Sensitivity in Children
}

\author{
(1) Ceyda Tuna Kırsaçlıoğlu1', (1) Gülin Hizal1, (1) Gülser Dinç22, (1) Seyhan Temtek Güner22, (1) Semih Sanda², \\ (1) Özden Üneri2
}

${ }^{1}$ Turkish Republic Health Ministry Ankara Child Health Diseases, Haematology Oncology Training and Research Hospital, Clinic of Pediatric Gastroenterology, Hepatology and Nutrition, Ankara, Turkey

2Turkish Republic Health Ministry Ankara Child Health Diseases, Haematology Oncology Training and Research Hospital, Clinic of Child and Adolescent Psychiatry, Ankara, Turkey

\begin{abstract} transglutaminase immunoglobulin (Ig) A and total IgA] and NCGS (anti-gliadin IgA/IgG antibodies). symptoms and anti-gliadin IgG positivity $(p=0.08)$.

Conclusion: There was no increase in the frequency of CD and NCGS in children with ADHD.

Keywords: Attention-deficit hyperactivity disorder, celiac disease, children, non-celiac gluten sensitivity
\end{abstract}

Aim: Dietary factors are considered one of the possible environmental risk factors for attention-deficit hyperactivity disorder (ADHD). The aim of this study was to demonstrate the relationship between ADHD and celiac disease (CD) and non-celiac gluten sensitivity (NCGS) in children.

Materials and Methods: In this prospective study, children with ADHD, aged 6-18 years, were asked about the presence of gastrointestinal symptoms and their relationship with gluten intake with a previously prepared questionnaire form. Also, they were screened for CD [anti-tissue

Results: Of the 117 children (76\% male), the mean age was $9.2 \pm 2.4$ years. Ninety-six patients $(82 \%)$ had no gastrointestinal complaints. There was no relationship between gluten intake and complaints in the patients who had constipation (12.8\%), recurrent abdominal pain (2.5\%), dyspeptic symptoms (1.7\%), and irritable bowel syndrome (0.8\%). None of the patients had anti-tissue transglutaminase IgA or IgG positivity. Only $1(0.8 \%)$ patient had anti-gliadin IgA, and $6(5.1 \%)$ patients had anti-gliadin IgG positivity. There was no relation between the presence of

\section{Introduction}

Wheat allergy (WA), celiac disease (CD) and non-celiac gluten sensitivity (NCCS) are the most common glutenrelated disorders (1-3). In recent years, the relationship between neuropsychiatric disorders and gluten ingestion such as CD and NCGS has been reported (3-6).

Attention-deficit hyperactivity disorder (ADHD) is characterized by hyperactive, inattentive and impulsive behavior and it is a common neuropsychiatric disorder in childhood. There is no consensus on the etiology of ADHD, but an interaction of genetic factors and environmental causes such as dietary factors have been considered as the main trigger factors. Also, the effect of elimination diets on the treatment of ADHD has been studied. The benefit of elimination of food additives from the diet has been reported in a minority of children with $A D H D$, but the role

\section{Address for Correspondence}

Ceyda Tuna Kırsaçlıoğlu, Turkish Republic Health Ministry Ankara Child Health Diseases, Haematology Oncology Training and Research Hospital, Clinic of Pediatric Gastroenterology, Hepatology and Nutrition, Ankara, Turkey

Phone: +90 5067054848 E-mail: ceytun@yahoo.com ORCID: orcid.org/0000-0002-3551-7267

Received: 15.07.2020 Accepted: 24.09.2020

${ }^{(\odot)}$ Copyright 2021 by Ege University Faculty of Medicine, Department of Pediatrics and Ege Children's Foundation The Journal of Pediatric Research, published by Galenos Publishing House. 
of other elimination diets such as gluten or casein-free diets and oligoantigenic diets on the improvement of ADHD was inconclusive in children $(7,8)$.

Depending on the hypothesis of the effect of diet on neurodevelopmental disorders, we aimed to evaluate the association of ADHD and the gluten-related disorders of both CD and NCGS in children.

\section{Materials and Methods}

The children who had been diagnosed as ADHD according to the criteria of "Diagnostic and Statistical Manual of Mental Disorders, Fourth Edition" in our child and adolescent psychiatry department were evaluated for CD and NCCS at our pediatric gastroenterology outpatient clinic between 07.12.2015 and 01.03.2016, prospectively. First of all, the patients and their parents were informed about the possible relationship between ADHD and gluten-related disorders and then they were asked if they would like to participate in the study. Subsequently, a previously designed informed consent form was given to the parents and their children (>12 years old). Children aged between 6-18 years old, diagnosed as ADHD and willing to participate in the study with a signed informed consent form were recruited into this study. Those children who had mental retardation, previously known $C D$, or refusing to give blood samples were excluded from the study.

The demographic findings of the patients, their medical history, their physical examination findings and anthropometric measurements were recorded. According to the body mass index (BMI) of the patients, overweight $(\geq 85 \%-<95 \%)$, obesity ( $\geq 95 \%)$ and underweight $(<5 \%)$ were defined. Those children who had height-for-age Z-score $<-2$ standard deviation were considered short stature $(9,10)$.

In order to determine NCGS, their gastrointestinal complaints were investigated with a previously prepared questionnaire form which consists of the following issues; abdominal pain, bloating, diarrhea, constipation, and abdominal distension. In the presence of any of these complaints in daily life, the relation to gluten intake was investigated. If they had a gluten-related symptoms, the following steps were planned; to give a gluten-free diet, to follow the patient for the presence of symptoms and, if there was an improvement within a month, to re-introduce gluten into the diet. If the symptoms occurred after re-introducing gluten, NCGS would be considered (11). Regardless of the presence of symptoms, anti-gliadin (AGA), immunoglobulin (Ig) A and IgG levels were studied in all patients.

All patients were screened for CD via anti-tissue transglutaminase IgA antibody and total IgA levels. In cases of low IgA levels for age, anti-tissue transglutaminase IgG antibody tests were studied. If the anti-tissue transglutaminase IgA or IgC antibody were positive, the following procedures were planned; to perform an upper gastrointestinal endoscopy, to take multiple biopsies from the small bowel; 4 biopsies from the second part of the duodenum and 2 biopsies from the duodenal bulb for histopathological examination in order to establish a biopsy-proven CD (12).

According to our laboratory data, the cut-off values of anti-tissue transglutaminase IgA and IgG, AGA IgA and IgG were lower than $18 \mathrm{U} / \mathrm{mL}$. Those values $>18 \mathrm{U} / \mathrm{mL}$ were accepted as a positive test.

\section{Statistical Analysis}

The data analysis was performed using the Statistical Package for Social Sciences software 17.0. Descriptive statistics, independent samples t-test, and the nonparametric Mann-Whitney $U$ test were used as appropriate, $p$-values $<0.05$ were considered statistically significant. The study was approved by the local ethics committee of our hospital (protocol number: 2015/073).

\section{Results}

A total of 117 children diagnosed as ADHD, with a mean age of $9.2 \pm 2.4$ years (age range of 6-17 years) were recruited into this study. Of these children, 89 (76\%) were male.

Ninety-six patients (82\%) had no gastrointestinal complaints. Fifteen (12.8\%) patients had constipation, 3 (2.5\%) had recurrent abdominal pain, 2 (1.7\%) had dyspeptic symptoms, and $1(0.8 \%)$ had irritable bowel syndrome. Of the 15 patients with constipation, 3 (2.5\%) had fecal incontinence. None of these symptoms were related to gluten intake ( $p>0.05)$.

The patients did not differ in terms of age and gender according to the presence of symptoms $(p=0.93, p=0.98$ respectively).

The anthropometric measurements revealed obesity in 5 (4.2\%) patients and underweight in 1 (0.8\%) patient according to age- and gender-appropriate BMI. Five $(4.2 \%)$ patients were stunted and two of these were also underweight.

None of the patients had anti-tissue transglutaminase IgA positivity. Seven (5.9\%) patients had IgA deficiency. Of these patients, none of them had anti-tissue transglutaminase IgG positivity.

Only $1(0.8 \%)$ patient had AGA IgA positivity (in titer 37.3 $\mathrm{U} / \mathrm{mL}), 6(5.1 \%)$ patients had AGA IgG positivity but all in low titers $(<50 \mathrm{U} / \mathrm{mL})$. 
Among those patients who had gastrointestinal symptoms, only $14.2 \%$ (3/21 patients) had AGA IgC positivity. Of these, 2 patients had functional constipation, and one patient had dyspeptic symptoms unrelated to gluten intake. There was no relation between the presence of these symptoms and AGA IgG positivity $(p=0.08)$.

\section{Discussion}

$C D$ is an immune-mediated inflammatory systemic disorder, triggered by gluten intake in genetically susceptible individuals. CD may present with classical form such as diarrhea, growth failure, but more frequently with nonclassical signs $(1,12)$. The neuropsychiatric problems such as depression, anxiety, behavioral and personality disorders, ataxia, migraine, epilepsy, and ADHD have been observed in $6.8 \%$ to $33.3 \%$ of children with $C D$ at presentation (3-6). Additionally, children with CD were found to have an increased risk (1.4-fold greater) for psychiatric disorders in the future (3). Recently, Kristensen et al. (13) reported high depression and anxiety scores in newly diagnosed adult CD patients and improvement after a minimum 1-year glutenfree diet.

There are limited studies that observe the relationship between ADHD and CD in children. The diagnose of ADHD had been found 1.2-fold higher in children with $C D$ than in the normal population (3). Niederhofer and Pittschieler (14) reported a high ADHD symptomatology score after the diagnosis of $C D$ and a significant decrease in ADHD score after 6 months gluten-free diet. Additionally, Niederhofer (15) reported a high frequency of CD (14.9\%) among children and adults with ADHD. On the other hand, Gungor et al. (16) reported $0.27 \%$ of the children with ADHD had biopsyproven $C D$ which is lower than the prevalence in healthy children $(0.47 \%)$ in our country (17). In our study, none of the patients had positive anti-tissue transglutaminase antibody and therefore CD. This may be due to the small size of our study population.

NCGS is a clinical condition, characterized with intestinal and extraintestinal symptoms which are triggered by gluten intake and resolve with gluten removal, in the absence of CD and WA $(1,2,18)$. The most common gastrointestinal symptoms are abdominal distension, bloating, diarrhea, and abdominal pain, followed by constipation, nausea, vomiting, and heartburn. The most common extra-intestinal symptoms are anxiety, lack of well-being, dizziness, and trouble in focusing $(1,2,18,19)$. In our study group, $17.9 \%$ of the children had gastrointestinal complaints, but none of these were related to gluten intake. The most common complaint was functional constipation (12.8\%) with a similar prevalence to healthy children in our country (20).
We did not investigate whether they had confusion, anxiety, or trouble in focusing because of their primary diagnosis.

Unfortunately, there is no consensus on a screening test for NCGS. In patients with NCGS, positivity of AGA IgC antibodies were found more frequently than in the general population but it still unclear that this is helpful in diagnosing NCGS (11). In healthy people, the frequency of AGA IgG antibody positivity is $2 \%$ to $8 \%$ (18). AGA IgA and IgC antibody were found in $7.7 \%$ and $56.4 \%$ of adults with NCGS, respectively and after gluten withdrawal, a disappearance of AGA IgG antibody was reported $(1,21)$. In our study, $0.8 \%$ of the patients had positive AGA IgA and $5.1 \%$ had positive AGA IgG antibody, which is not more frequent than in the general population.

ADHD is a common neuropsychiatric disorder in childhood, characterized by hyperactive, inattentive and impulsive behavior. There is no consensus on the etiology of ADHD, but interactions of genetic and environmental factors are thought to be the main triggering factors. Diet is thought to be related to ADHD symptoms $(7,8)$. In previous studies, it was reported that a minority of children with ADHD benefit from the exclusion of food additives, but the role of elimination diets such as a gluten-free diet, a caseinfree diet, or oligoantigenic diets was inconclusive (8). It was reported that approximately $33 \%$ of children with ADHD may respond to dietary intervention in a meta-analysis (22). The children with ADHD who ate a well-balanced diet had lower inattention score, than those who prefer the sweetened desserts and junk food (23). In the metaanalysis which reviewed the randomized controlled trials for exclusion and challenge of colorants, improvement of hyperactivity in $8 \%$ of patients with ADHD was observed (22). Additionally, a minority of children with ADHD benefit from the exclusion of food additives (7). The oligoantigenic diet, which consists of milk and dairy products, egg, soy, peanuts, wheat and seafood elimination, demonstrated a significant decrease in hyperactive behaviors in $64 \%$ of children in randomized controlled trials (24). In light of these findings that demonstrated the effect of dietary gluten intake on ADHD, we investigated the relationship between ADHD and gluten-related disorders, however we could not find any relationship.

\section{Study Limitations}

The major limitation of our study is the age range (only children) and the small number in the study population. Though wheat and wheat-based foods play an important role in our traditional eating pattern, the intense ingestion of gluten in daily life leads to difficulty in revealing the exact relationship between symptoms and gluten intake, 
especially in children with ADHD.

\section{Conclusion}

Although this study demonstrates that CD and NCGS are not common in children with ADHD, larger sized studies are needed.

\section{Ethics}

Ethics Committee Approval: The study was approved by the local ethics committee of our hospital (protocol number: 2015/073).

Informed Consent: Children aged between 6-18 years old, diagnosed as ADHD and willing to participate in the study with a signed informed consent form were recruited into this study.

Peer-review: Externally and internally peer-reviewed.

\section{Authorship Contributions}

Concept: S.S., C.T.K., G.D., Design: C.T.K., G.D., G.H., Ö.Ü., Data Collection or Processing: C.T.K., G.D., G.H., S.S., S.T.G., Analysis or Interpretation: C.T.K., G.D., S.T.G., Literature Search: C.T.K., S.S., S.T.G., Writing: C.T.K., G.H., G.D., Ö.Ü.

Conflict of Interest: There is no conflict of interest is declared by the authors.

Financial Disclosure: The authors declare that this study received no financial support.

\section{References}

1. Meijer CR, Shamir R, Mearin ML. Coeliac disease and noncoeliac gluten sensitivity. J Pediatr Gastroenterol Nutr 2015; 60:429-32.

2. Sapone A, Bai JC, Ciacci C, et al. Spectrum of gluten-related disorders: consensus on new nomenclature and classification. BMC Med 2012; 10:13.

3. Butwicka A, Lichtenstein P, Frisen L, et al. Celiac disease is associated with childhood psychiatric disorders: A populationbased study, I Pediatr 2017; 184:87-93.

4. Isikay S, Kocamaz H. The neurological face of celiac disease. Arq Gastroenterol 2015; 52:167-70.

5. Sel Genc C, Aksoy E, Aksoy A, Yuksel D, Ozbay F. Neurological manifestations of atypical celiac disease in childhood. Acta Neurol Belg 2017; 117:719-27.

6. Diaconu G, Burlea M, Grigore I, Anton DT, Trandafir LM. Celiac disease with neurologic manifestations in children. Rev Med Chir Soc Med Nat lasi 2013; 117:88-94.

7. Ly V, Bottelier M, Hoekstra PJ, Arias Vasquez A, Buitelaar JK, Rommelse NN. Elimination diets' efficacy and mechanisms in attention deficit hyperactivity disorder and autism spectrum disorder. Eur Child Adolesc Psychiatry 2017; 26:1067-79.
8. Nigg JT, Holton K. Restriction and elimination diets in ADHD treatment. Child Adolesc Psychiatr Clin N Am 2014; 23:937-53.

9. Kumar S, Kelly AS. Review of childhood obesity: from epidemiology, etiology, and comorbidities to clinical assessment and treatment. Mayo Clin Proc 2017; 92:251-65.

10. Mehta NM, Corkins MR, Lyman B, et al. American Society for Parenteral, Enteral Nutrition Board of Directors: Defining pediatric malnutrition: a paradigm shift toward etiology-related definitions. J Parenter Enteral Nutr 2013; 37:460-81.

11. Catassi C, Elli L, Bonaz B, et al. Diagnosis of non-celiac gluten sensitivity (NCCS): The Salerno Experts' Criteria. Nutrients 2015; 7:4966-77.

12. Husby S, Koletzko S, Korponay-Szabo IR, et al. European Society for Pediatric Gastroenterology, Hepatology, and Nutrition guidelines for the diagnosis of coeliac disease. I Pediatr Gastroenterol Nutr 2012; 54:136-60.

13. Kristensen VA, Valeur I, Brackmann S, Jahnsen J, Brunborg C, Tveito K. Attention deficit and hyperactivity disorder symptoms respond to gluten-free diet in patients with coeliac disease. Scand I Gastroenterol 2019; 54:571-6.

14. Niederhofer $\mathrm{H}$, Pittschieler K. A preliminary investigation of ADHD symptoms in persons with celiac disease. I Atten Disord 2006; 10:200-4.

15. Niederhofer $\mathrm{H}$. Association of attention-deficit/hyperactivity disorder and celiac disease: a brief report. Prim Care Companion CNS Disord 2011; 13:PCC.10br01104. doi: 10.4088/PCC.10br01104.

16. Gungor S, Celiloglu OS, Ozcan OO, Raif SG, Selimoglu MA. Frequency of celiac disease in attention-deficit/hyperactivity disorder. J Pediatr Gastroenterol Nutr 2013; 56:211-4.

17. Dalgic B, Sari S, Basturk B, et al. Prevalence of celiac disease in healthy Turkish school children., Am I Gastroenterol 2011: 106:1512-7.

18. Czaja-Bulsa G. Non coeliac gluten sensitivity - a new disease with gluten intolerance. Clin Nutr 2015; 34:189-94.

19. Francavilla R, Cristofori F, Verzillo L, et al. Randomized doubleblind placebo-controlled crossover trial for the diagnosis of non-celiac gluten sensitivity in children. Am / Gastroenterol 2018; 113:421-30.

20. Uğuralp S, Karaoğlu L, Karaman A. Frequency of enuresis, constipation and enuresis association with constipation in a group of school children aged 5-9 years in Malatya, Turkey. Turk J Med Sci 2003; 33:315-20.

21. Volta $U$, Tovoli F, Cicola $R$, et al. Serological tests in gluten sensitivity (nonceliac gluten intolerance). J Clin Gastroenterol 2012; 46:680-5.

22. Nigg JT, Lewis K, Edinger T, Falk M. Meta-analysis of attentiondeficit/hyperactivity disorder or attention-deficit/hyperactivity disorder symptoms, restriction diet, and synthetic food color additives. J Am Acad Child Adolesc Psychiatry 2012; 51:86-97.

23. Park S, Cho SC, Hong YC, et al. Association between dietary behaviors and attention-deficit/hyperactivity disorder and learning disabilities in school-aged children. Psychiatry Res 2012; 198:468-76.

24. Pelsser LM, Frankena K, Toorman J, et al. Effects of a restricted elimination diet on the behaviour of children with attentiondeficit hyperactivity disorder (INCA study): A randomised controlled trial. Lancet 2011; 377:494-503. 